\title{
Oral temazepam causing anxiety
}

\author{
SAAD President's Advisory Group; Nigel D. Robb, Senior Lecturer \\ in Sedation in Relation to Dentistry/Honorary Consultant in \\ Restorative Dentistry, Glasgow Dental Hospital and School
}

Conscious sedation is one of the most highly regulated areas in dentistry. However, despite the plethora of standards and guidelines, there is little advice relating to the use of 'premedication' either alone or in combination with conscious sedation techniques.

We have become aware during postgraduate courses and from dental defence organisations of the practice of giving patients a small but significant dose of oral temazepam at the surgery as a way of reducing anxiety. The term 'anxiolysis' has been used to describe this practice; the implication being that the rules relating to conscious sedation are therefore not relevant. Considering the doses of temazepam which have been used, we beg to differ, particularly since it has emerged that some individuals engaging in this practice have not undergone any training in conscious sedation. We consider that patients may be at risk.

There are two challenges. First, there is no clear distinction between premedication and conscious sedation and second, the oral administration of drugs is often considered safer than intravenous (IV) administration. Premedication is normally taken by the patient prior to arrival at the dental surgery and is sometimes a precursor to another anxiety management technique, for example intravenous sedation. In contrast, conscious sedation normally involves the administration of oral, intranasal, inhalational or intravenous drugs in the surgery by a suitably trained and experienced sedationist. The great advantage of administering sedatives intravenously or by inhalation is that they can be titrated against the individual patient's response. This is not possible with drugs given orally because of the long latent period between administration and effect.

Current guidance from the Standing Committee on Sedation for Dentistry of the Faculty of Dental Surgery of the Royal College of Surgeons of England, published jointly with the Royal College of Anaesthetists, states that when orally administered conscious sedation is used the sedationist must be competent in the use of intravenous sedation. This is to ensure prompt and effective management in the event of accidental oversedation or other sedation-related complications. This might include the insertion of an intravenous cannula and the administration of the benzodiazepine antagonist flumazenil. It is sometimes overlooked that the monitoring and staffing requirements for oral sedation are identical to those for the administration of intravenous sedation and that all patients who receive oral sedation should be cannulated as soon as they are sufficiently sedated.

The situation is further complicated by the British National Formulary, which specifies a dose of $20-40 \mathrm{mg}$ of temazepam for premedication, whilst dental texts written at a time when temazepam was considered to be the oral sedative of choice recommend doses of 10-40 $\mathrm{mg}$. One of the probable reasons for the disparity in the range of recommended doses is the unpredictability of the effects of orally administered benzodiazepines. In some patients $40 \mathrm{mg}$ of temazepam produces minimal clinical effects whilst in others as little as $10 \mathrm{mg}$ produces profound sedation. There is no known method of identifying which patients are susceptible and which are resistant to benzodiazepines. Slow and careful titration is only way to achieve the optimum level of sedation.

The Board of the Society for the Advancement of Anaesthesia in Dentistry (SAAD) is concerned by the use of oral temazepam as 'premedication' and does not consider that calling oral sedation 'anxiolysis' contributes anything to ensuring patient safety. We believe that it is simply either misguided or an attempt to circumvent the current guidance on oral sedation. ${ }^{1}$

There is a clearly a need for enhanced clarification and guidance, including more detailed information about the doses of oral benzodiazepines which might be given as premedication by those without training and experience in intravenous conscious sedation. However, we would urge caution when prescribing any benzodiazepine as premedication, as this route of administration is associated with a longer duration of action and less predictable effects than intravenously administered midazolam.

It is also important that those who are prescribed premedicant doses of benzodiazepines receive appropriate instructions for the postoperative period. Driving whilst under the influence of these agents may be a criminal offence. The sedative effects of a one-off administration will be greater than the effects of the same dose in a regular user, and so patients should receive similar warnings to those given for intravenous conscious sedation.

We are concerned that unless the guidance in this area of practice is clarified, there is the potential for patients to suffer harm either by being sedated without the appropriate level of care being available or because of the impairment of judgement in the postoperative period. Any adverse reports would undoubtedly have serious consequences for the use of conscious sedation, which we consider to be an important element in the provision of safe and effective pain and anxiety control in dentistry.

DOI: $10.1038 /$ sj.bdj.2010.630

1. Royal College of Anaesthetists and Faculty of Dental Surgery of the Royal College of Surgeons of England. Standards for conscious sedation in dentistry: alternative techniques. A Report from the Standing Committee on Sedation for Dentistry. London: Royal College of Surgeons of England/Royal College of Anaesthetists, 2007. http://www.dstg.co.uk/files/teaching/scsdat-2007.pdf 